\title{
Adaptive and Fault-Tolerant Routing with $100 \%$ Node Utilization for Mesh Multicomputer
}

\author{
Sheng-De Wang* and Ming-Jer Tsai \\ Department of Electrical Engineering, National Taiwan University, \\ Taipei 106, TAIWAN
}

\begin{abstract}
In this paper, we propose an adaptive and deadlock-free routing algorithm to tolerate irregular faulty patterns using two virtual channels per physical link. It can improve the node utilization up to $100 \%$. When a node becomes faulty or recovered, the central control unit constructs a directed path graph which is used for generating the intermediate nodes of the message path. Thus a message can be transmitted from sources or to destinations within faulty blocks via a set of "intermediate nodes". Our method requires the global failure information if the central control unit is not available.
\end{abstract}

\section{Introduction}

Message routing achieves inter-node communication in large-scale parallel computers. In a concurrent multicomputer, wormhole switching [9] is widely used; however, it suffers the deadlock problem when not well-designed in a fault environment. Thus, a reliable routing algorithm is supposed to be deadlock-free and fault-tolerant.

Glass and $\mathrm{Ni}$ [6] have proposed a partially-adaptive routing algorithm to tolerate $n-1$ faults in an $n$-dimensional mesh based on the turn model. In an $N \times N$ 2-dimensional mesh, Cunningham and Avresky [4] can improve the performance and provide fault tolerance for up to $N-1$ faults. Besides, Hadas and Brandt [7] have proposed an originalbased routing algorithm to tolerate square faulty blocks. When a fault arises, their method has a hot-spot effect and will non-minimally route a message even if the message does not encounter any square faulty block.

Moreover, Linder and Harden [8] have proposed a fullyadaptive and fault-tolerant routing algorithm based on the concept of virtual interconnection networks. However, it requires exponential number of virtual channels per physical link and tolerates small number of faults. Dally and Aoki

\footnotetext{
*Corresponding author. E-mail: sdwang@hpc.ee.ntu.edu.tw.
}

[5] have proposed a dynamic algorithm to remove cycles from packet wait-for graph instead of channel dependency graphs. Thereby, the virtual channel utilization can be considerably improved. In their algorithm, the number of faults tolerated and virtual channels used depends on the location of faults.

Using three virtual channels per physical link, Chien and Kim [3] have proposed a planar-adaptive routing algorithm to tolerate disconnected faulty blocks with distance of no less than two in at least one dimension. Using extra four virtual channels per physical link, Boppana and Chalasani [1] can enhance a fully-adaptive algorithm to tolerate disconnected faulty blocks with distance of no less than two in at least one dimension. Using three virtual channels per physical link, the algorithm [2] presented by Boura and Das provides full-adaptivity and fault-tolerance. It can tolerate disconnected faulty blocks with distance of at least three.

In addition, Su and Shin [10] have proposed an adaptive routing algorithm to tolerate disconnected faulty blocks with distance of three or more in at least one dimension using only two virtual channels per physical link which is so far the smallest. However, their algorithm can possibly reach a deadlock and has been improved to tolerate disconnected faulty blocks with distance of no less than two in at least one dimension [11].

As was stated above, many adaptive routing algorithms $[1,3,10]$ are designed to tolerate a large number of faults by introducing rectangular faulty blocks. Thus, some good nodes are regarded as faulty ones and are prohibited from interchanging messages with the other good nodes. It implies that the node utilization may drastically degrade when the faulty nodes densely distribute in certain particular patterns. In this paper, using two virtual channels per physical link, we develop an adaptive and deadlock-free routing algorithm, by which two good nodes can communicate with each other if there is at least one path between them. Notation used in this paper are summarized in Table 1, where two nodes are connected if there is a link between them, and two sets $S_{i}$ and $S_{j}$ are connected if there is a node $A \in S_{i}$ and a node $B \in S_{j}$ such that nodes $A$ and $B$ are connected 
Table 1. Summary of Notation

$V I N_{i} \quad$ the virtual interconnection network $i(i=$ $1,2)$.

$V C_{i, j}$ the virtual channel in dimension $i$ of $V I N_{j}$.

$\operatorname{num}\left(V C_{i, j}\right)$ the channel number of virtual channel $V C_{i, j}$.

$N_{S} \quad$ the source node.

$N_{D}$ the destination node.

$N_{C}$ the current node.

$N_{I}$ the intermediate node.

$N_{R}$ the node receives the message from an intermediate node.

$B_{i} \quad$ the faulty block $i$.

$M_{0}$ the interconnection network outside all faulty blocks.

$M_{i} \quad$ the sub-mesh $i(i>0)$ contained in a faulty block.

$S_{-1} \quad$ a set consists of faulty nodes.

$S_{i} \quad$ a set consists of good nodes in $M_{i}(i \geq 0)$.

$V_{i} \quad$ vertex $i$ in the directed path graph.

$G_{i, j} \quad$ a set consists of the entry $\left(w_{i}, \operatorname{dim}, \operatorname{dir}\right)$, where $w_{i} \in S_{i}$ is an intermediate node to enter $S_{j}\left(S_{i}\right.$ and $S_{j}$ are connected) for the message with header in $M_{i}$, and the message is routed to $w_{i}$ via $V I N_{d i r}$ if $S_{i} \neq S_{0}$, then is routed out from $w_{i}$ via $V C_{d i m, d i r}$.

$T_{i, j} \quad$ a set consists of the entry $\left(w_{i}, \operatorname{dim}, \operatorname{dir}\right)$, where $w_{i} \in S_{i}$ is an intermediate node to reach $N_{D} \in S_{j}\left(S_{i}\right.$ may or may not connect to $S_{j}$ ) for the message with header in $M_{i}$, and the message is routed to $w_{i}$ via $V I N_{d i r}$ if $S_{i} \neq S_{0}$, then is routed out from $w_{i}$ via $V C_{d i m, d i r}$.

nodes.

The rest of this paper is organized as follows. In the next section, we first present a method to construct the directed path graph which is used to generate the intermediate node of the message path. In section 3 , we propose an adaptive and deadlock-free routing algorithm to tolerate irregular faulty patterns using two virtual channels per physical link. In section 4, we conclude this paper.

\section{Construct the directed path graph}

In this section, algorithm 2.1 is presented to construct the directed path graph which is used to generate the message path in the next section. Before describing it, Definitions 1 and 2 are needed.
Definition 1 (Safe/Unsafe Node) For an n-dimensional mesh, a good node is called an unsafe node if it connects to at least two faulty/unsafe nodes, and is called a safe node otherwise.

Definition 2 (Ancestor, Common Ancestor, Least Common Ancestor) In the directed path graph, if there is a directed path from vertex $A$ to vertex $B$, then vertex $A$ is called an ancestor of vertex $B$. If vertex $A$ is an ancestor of both vertices $B$ and $C$, then vertex $A$ is called the common ancestor of vertices $B$ and $C$. For two vertices $B$ and $C$, common ancestor $A$ is called the least common ancestor if no other common ancestor is located in each directed shortest path from vertex $A$ to vertex $B$ and in each directed shortest path from vertex $A$ to vertex $C$.

Algorithm 2.1 /* When a node becomes faulty or recovered, algorithm 2.1 is executed by the central control unit. And, the central control unit will send $T_{i, *}$ to all nodes in $S_{i}$ after algorithm 2.1 is completed. */

1 Let the directed path graph be an empty graph.

2 Add vertex $V_{0}$ to the directed path graph and set $x$ to 1 .

3 For all faulty blocks $B_{1}$ to $B_{p}$ do $/ *$ construct the directed path graph, and compute $G_{k, j}$ and $G_{j, k}$ for each directed edge $\left(V_{k}, V_{j}\right)$.*/

3.1 Find sub-meshes $M_{x}, M_{x+1}, \cdots, M_{x+q-1}$ in $B_{i}$ such that

(1) Each good node in $B_{i}$ belongs to exactly one $M_{j}(x \leq j \leq x+q-1)$.

(2) For each $M_{j}(x \leq j \leq x+q-1)$, the number of faulty nodes in $M_{j}$ is less than the dimension of $M_{j}$.

(3) For each $M_{j}(x \leq j \leq x+q-1)$, there exists $M_{0}=M_{a_{1}}, M_{a_{2}}, \cdots, M_{a_{b}}=M_{j}$ such that (1) $S_{a_{c}}$ and $S_{a_{c+1}}$ are connected and (2) $a_{c}<a_{c+1}$, where $M_{a_{2}}, M_{a_{3}}, \cdots, M_{a_{b}}$ are all in $B_{i}$.

3.2 For $j=x$ to $x+q-1$ do

3.2.1 Add vertex $V_{j}$ to the directed path graph.

3.2.2 For each $S_{k}(0 \leq k<j)$ connected to $S_{j}$ do

3.2.2.1 Add a directed edge $\left(V_{k}, V_{j}\right)$ to the directed path graph.

3.2.2.2 Let $G_{k, j}$ and $G_{j, k}$ be empty sets.

3.2.2.3 For each pair of connected nodes $w_{k} \in S_{k}$ and $w_{j} \in S_{j}$ do

3.2.2.3.1 $G_{k, j}=G_{k, j} \cup\left(w_{k}, \operatorname{dim}, 1\right)$. 3.2.2.3.2 $G_{j, k}=G_{j, k} \cup\left(w_{j},-\operatorname{dim}, 2\right)$. 
I* $\operatorname{dim}$ denotes the directed dimension where the link from node $w_{k}$ to node $w_{j}$ is located. */

$3.3 x=x+q$.

4 For $j=0$ to $x-1$ do $/ *$ compute $T_{j, k}$ for $0 \leq j \leq$ $x-1,0 \leq k \leq x-1$, and $j \neq k$, where $x$ is the number of vertices in the directed path graph. */

4.1 For $k=0$ to $x-1(k \neq j)$ do

4.1.1 If vertex $V_{k}$ is an ancestor of vertex $V_{j}$ in the directed path graph, then

4.1.1.1 $T_{j, k}$ is set to $\emptyset$.

4.1.1.2 For each directed shortest path $P_{u p}$ from vertex $V_{k}$ to vertex $V_{j}$ do.

4.1.1.2.1 Let $\left(V_{a}, V_{j}\right)$ be a directed edge in $P_{u p}$. Then, $T_{j, k}$ is set to $T_{j, k} \cup G_{j, a}$.

4.1.2 Else if vertex $V_{j}$ is an ancestor of vertex $V_{k}$ in the directed path graph, then

4.1.2.1 $T_{j, k}$ is set to $\emptyset$.

4.1.2.2 For each directed shortest path $P_{\text {down }}$ from vertex $V_{j}$ to vertex $V_{k}$ do

4.1.2.2.1 Let $\left(V_{j}, V_{a}\right)$ be a directed edge in $P_{\text {down }}$. Then, $T_{j, k}$ is set to $T_{j, k} \cup G_{j, a}$.

4.1.3 Else if vertices $V_{j}$ and $V_{k}$ has no common ancestor in the directed path graph, then $T_{j, k}$ is set to $\emptyset$.

\subsubsection{Else}

4.1.4.1 $T_{j, k}$ is set to $\emptyset$.

4.1.4.2 For each least common ancestor $V_{l c a}$ of vertices $V_{j}$ and $V_{k}$ do

4.1.4.2.1 For each directed shortest path $P_{u p}$ from vertex $V_{l c a}$ to vertex $V_{j}$ do

4.1.4.2.1.1 Let $\left(V_{a}, V_{j}\right)$ be a directed edge in $P_{u p}$. Then, $T_{j, k}$ is set to $T_{j, k} \cup$ $G_{j, a} \cdot \square$

An example is shown in Figure 1(a), where 36 faulty nodes spread over a $21 \times 21$ mesh. Based on Definition 1 , three faulty blocks $B_{1}, B_{2}$, and $B_{3}$ are formed by the deactivating method. After algorithm 2.1 is completed, we have

$1 B_{1}$ finds sub-mesh $M_{1}, B_{2}$ finds sub-mesh $M_{2}$, and $B_{3}$ finds sub-meshes $M_{3}$ and $M_{4}$ due to step 3.1.

2 The directed path graph is constructed as shown in Figure 1(b) due to step 3.2.1.
Table 2. $T_{j, k}$ obtained for the injured mesh shown in Figure 1(a).

\begin{tabular}{||c||c|c|c|c|c||}
\hline \hline$j \backslash k$ & 0 & 1 & 2 & 3 & 4 \\
\hline \hline 0 & - & $G_{0,1}$ & $G_{0,2}$ & $G_{0,3}$ & $G_{0,3}$ \\
\hline \hline 1 & $G_{1,0}$ & - & $G_{1,0}$ & $G_{1,0}$ & $G_{1,0}$ \\
\hline \hline 2 & $G_{2,0}$ & $G_{2,0}$ & - & $G_{2,0}$ & $G_{2,0}$ \\
\hline \hline 3 & $G_{3,0}$ & $G_{3,0}$ & $G_{3,0}$ & - & $G_{3,4}$ \\
\hline \hline 4 & $G_{4,3}$ & $G_{4,3}$ & $G_{4,3}$ & $G_{4,3}$ & - \\
\hline \hline
\end{tabular}

3 For each directed edge $\left(V_{k}, V_{j}\right)$ in the directed path graph, $G_{k, j}$ and $G_{j, k}$ is obtained due to step 3.2.2, where $G_{0,1}=\{((6,16),-1,1),((7,16),-1,1),((8,-16)$,$1,1),((9,15),-0,1),((9,14),-0,1),((9,13),-0,1)\}, G_{1,0}=$ $\{((6,15), 1,2), \quad((7,15), 1,2), \quad((8,15), 1,2), \quad((8,15), 0,2)$, $((8,14), 0,2),((8,13), 0,2)\}, G_{0,2}=\{((11,10),-1,1)$, $((12,10),-1,1)\}, G_{2,0}=\{((11,9), 1,2),((12,9), 1,2)\}$, $G_{0,3}=\{((3,8),-1,1)\}, G_{3,0}=\{((3,7), 1,2)\}, G_{3,4}=$ $\{((3,7),-1,1)\}, G_{4,3}=\{((3,6), 1,2)\}$.

$4 T_{j, k}(0 \leq j \leq 4,0 \leq k \leq 4, j \neq k)$ is obtained as shown in Table 2 due to step 4 , where $T_{j, 0}$ is set to $G_{j, 0}$ for $1 \leq j \leq 3$ and $T_{4,3}$ is set to $G_{4,3}$ due to step 4.1.1, $T_{0, k}$ is set to $G_{0, k}$ for $1 \leq k \leq 3$ and $T_{3,4}$ is set to $G_{3,4}$ due to step 4.1.2, $T_{j, k}(j \neq k)$, excluding $T_{3,4}$, is set to $G_{j, 0}$ for $1 \leq j \leq 3,1 \leq k \leq 4$ and $T_{4, k}$ is set to $G_{4,3}$ for $k=1,2$ due to step 4.1.4.

Another example is shown in Figure 2(a), where 2 faulty blocks $B_{1}$ and $B_{2}$ form in $21 \times 21$ injured mesh. After algorithm 2.1 is completed, we have

$1 B_{1}$ finds sub-meshes $M_{1} \sim M_{7}$, and $B_{2}$ finds submeshes $M_{8} \sim M_{9}$.

2 The directed path graph is constructed as shown in Figure 2(b).

3 For each directed edge $\left(V_{k}, V_{j}\right)$ in the directed path graph, $G_{k, j}$ and $G_{j, k}$ is obtained.

$4 T_{j, k}(0 \leq j \leq 9,0 \leq k \leq 9)$ is obtained as shown in Table 3.

In step 3.1, each faulty block $B_{i}$ can find sub-meshes by a deterministic algorithm. Thus, our method requires the global failure information if the central control unit is not available. 


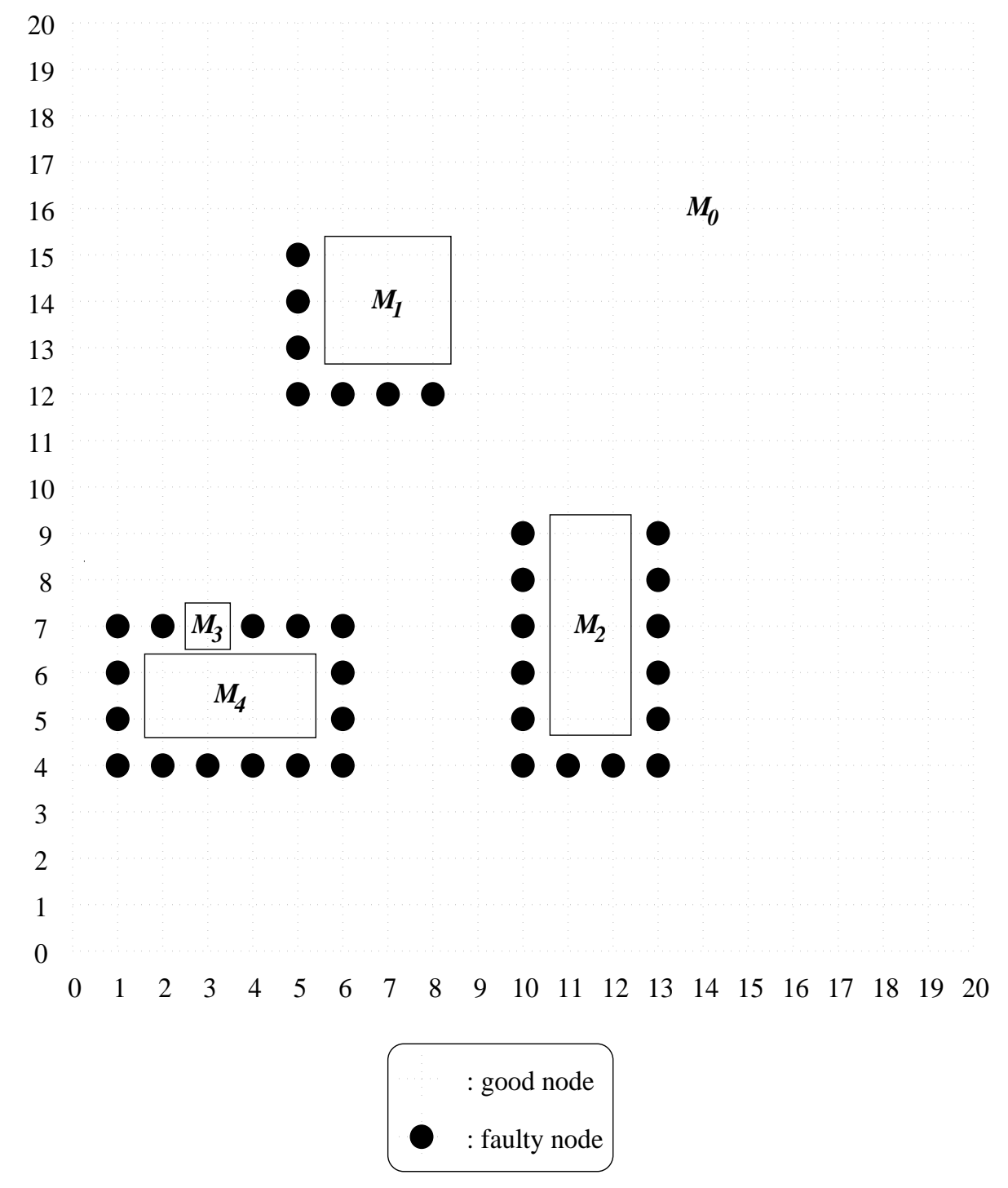

(a)

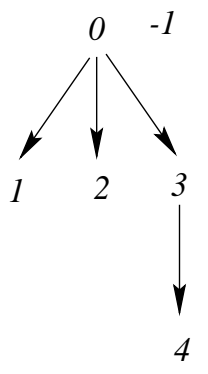

(b)

Figure 1. (a) shows a $21 \times 21$ injured mesh, where 3 faulty blocks $B_{1} \sim B_{3}$ are formed by Definition 1, and $M_{0} \sim M_{4}$ are decided after the completion of algorithm 2.1. (b) shows the directed path graph constructed by algorithm 2.1. 
Table 3. $T_{j, k}$ obtained for the injured mesh shown in Figure 2(a).

\begin{tabular}{|c|c|c|c|c|c|}
\hline \multirow{2}{*}{$j \backslash k$} & $\overline{0}$ & $\overline{1}$ & 2 & 3 & $\overline{4}$ \\
\hline & 5 & 6 & 7 & 8 & 9 \\
\hline \multirow{2}{*}{0} & - & $\overline{\bar{G} G_{0,1}}$ & $\overline{\bar{G} G_{0,2}}$ & $\overline{G_{0,1} \cup G_{0,2}}$ & $\overline{\bar{G} G_{0,4}}$ \\
\hline & $G_{0,2} \cup G_{0,4}$ & $G_{0,6}$ & $G_{0,7}$ & $\emptyset$ & $G_{0,9}$ \\
\hline \multirow{2}{*}{1} & $\overline{G_{1,0}}$ & - & $\overline{\bar{G} G_{1,2}}$ & $\overline{G_{1,3}}$ & $\overline{\bar{G}} G_{1,4}$ \\
\hline & $G_{1,2} \cup G_{1,3} \cup G_{1,4}$ & $G_{1,4}$ & $G_{1,2}$ & $\emptyset$ & $G_{1,0}$ \\
\hline \multirow{2}{*}{2} & $\overline{G_{2,0}}$ & $\overline{G_{2,1}}$ & - & $\overline{G_{2,3}}$ & $\overline{\overline{G_{2,0} \cup G_{2,1}}}$ \\
\hline & $G_{2,5}$ & $G_{2,0} \cup G_{2,1}$ & $G_{2,7}$ & $\emptyset$ & $G_{2,0}$ \\
\hline \multirow{2}{*}{3} & $G_{3,1} \cup G_{3,2}$ & $G_{3,1}$ & $G_{3,2}$ & - & $G_{3,1}$ \\
\hline & $G_{3,5}$ & $G_{3,1}$ & $G_{3,5}$ & $\emptyset$ & $G_{3,1} \cup G_{3,2}$ \\
\hline \multirow{2}{*}{4} & $G_{4,0}$ & $G_{4,1}$ & $G_{4,0} \cup G_{4,1}$ & $G_{4,1}$ & - \\
\hline & $G_{4,5}$ & $G_{4,6}$ & $G_{4,5} \cup G_{4,6}$ & $\emptyset$ & $G_{4,0}$ \\
\hline \multirow{2}{*}{5} & $\overline{G_{5,2} \cup G_{5,4}}$ & $\overline{\bar{G}_{5,2} \cup G_{5,3} \cup G_{5,4}}$ & $\overline{\overline{G_{5,2}}}$ & 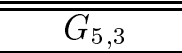 & $\overline{G_{5,4}}$ \\
\hline & - & $G_{5,4}$ & $G_{5,7}$ & $\emptyset$ & $G_{5,2} \cup G_{5,4}$ \\
\hline \multirow{2}{*}{6} & $G_{6,0}$ & $G_{6,4}$ & $G_{6,0} \cup G_{6,4}$ & $G_{6,4}$ & $G_{6,4}$ \\
\hline & $G_{6,4}$ & - & $G_{6,7}$ & $\emptyset$ & $G_{6,0}$ \\
\hline \multirow{2}{*}{7} & $G_{7,0}$ & $\overline{G_{7,2}}$ & $\overline{G_{7,2}}$ & $\overline{G_{7,5}}$ & 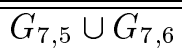 \\
\hline & $G_{7,5}$ & $G_{7,6}$ & - & $\emptyset$ & $G_{7,0}$ \\
\hline \multirow{2}{*}{8} & $\bar{\emptyset}$ & $\bar{\emptyset}$ & $\bar{\emptyset}$ & $\bar{\emptyset}$ & $\bar{\emptyset}$ \\
\hline & $\emptyset$ & $\emptyset$ & $\emptyset$ & - & $\emptyset$ \\
\hline \multirow{2}{*}{9} & $\overline{G_{9,0}}$ & $\overline{\bar{G} G_{9,0}}$ & $\overline{\bar{G} G_{9,0}}$ & $\overline{\bar{G} G_{9,0}}$ & $\overline{G_{9,0}}$ \\
\hline & $G_{9,0}$ & $G_{9,0}$ & $G_{9,0}$ & $\emptyset$ & - \\
\hline
\end{tabular}

\section{Adaptive and fault-tolerant routing with $100 \%$ node utilization}

In this section, algorithm 3.1 is proposed to route a message using two virtual channels per physical link. It uses Glass and Ni's algorithm [6] to route the message inside a faulty block, and uses algorithm RRFB2 [11] to route the message outside a faulty block. It is capable of tolerating irregular faulty patterns by transmitting the message from sources or to destinations within faulty blocks via multiple "intermediate nodes". In algorithm 3.1, the message header format is (header. $N_{D}$, header. $N_{I}$, header.dim, header.dir). header. $N_{D}$ records the address of $N_{D}$, header. $N_{I}$ records the address of $N_{I}$, header.dim records the directed dimension along which the message is routed out from $N_{I}$, and header.dir records the virtual interconnection network VIN $N_{\text {header.dir }}$ used to route the message from $N_{C}$ to $N_{R}$ if $N_{C} \notin S_{0}$. To allow clearer exposition, we assume $N_{S} \in S_{p}, N_{C} \in S_{q}, N_{D} \in S_{r}$ in algorithm 3.1.

Algorithm 3.1 /* Assume $N_{S} \in S_{p}, N_{C} \in S_{q}$, and $N_{D} \in$ $S_{r} *$ /

1 If $N_{C}=N_{D}$, then exit.
2 Else if $N_{C}=N_{I}$, then the message is routed via $V C_{\text {header.dim, header.dir. }}$.

3 Else

3.1 If $\left(N_{C}=N_{S}\right)$ or $\left(N_{C}=N_{R}\right)$, then Set_Message_Header.

3.2 If $S_{q}=S_{r}$, then

3.2.1 If $N_{C} \in S_{0}$, then route the message to $N_{D}$ via $V I N_{1}$ and $V I N_{2}$ by an adaptive deadlock-free routing algorithm in [10].

3.2.2 Else route the message to $N_{D}$ via $V I N_{\text {header.dir }}$ by an adaptive deadlock-free routing algorithm in [6].

3.3 Else

3.3.1 If $N_{C} \in S_{0}$, then route the message to $N_{I}$ via $V I N_{1}$ and $V I N_{2}$ by an adaptive deadlock-free routing algorithm in [10].

3.3.2 Else route the message to $N_{I}$ via $V I N_{\text {header.dir }}$ by an adaptive deadlock-free routing algorithm in [6].

Set_Message_Header 1 If $\left(N_{C}=N_{S}\right)$ and $\left(T_{p, r}=\emptyset\right.$ or $\left.N_{D} \in S_{-1}\right)$, then exit. 


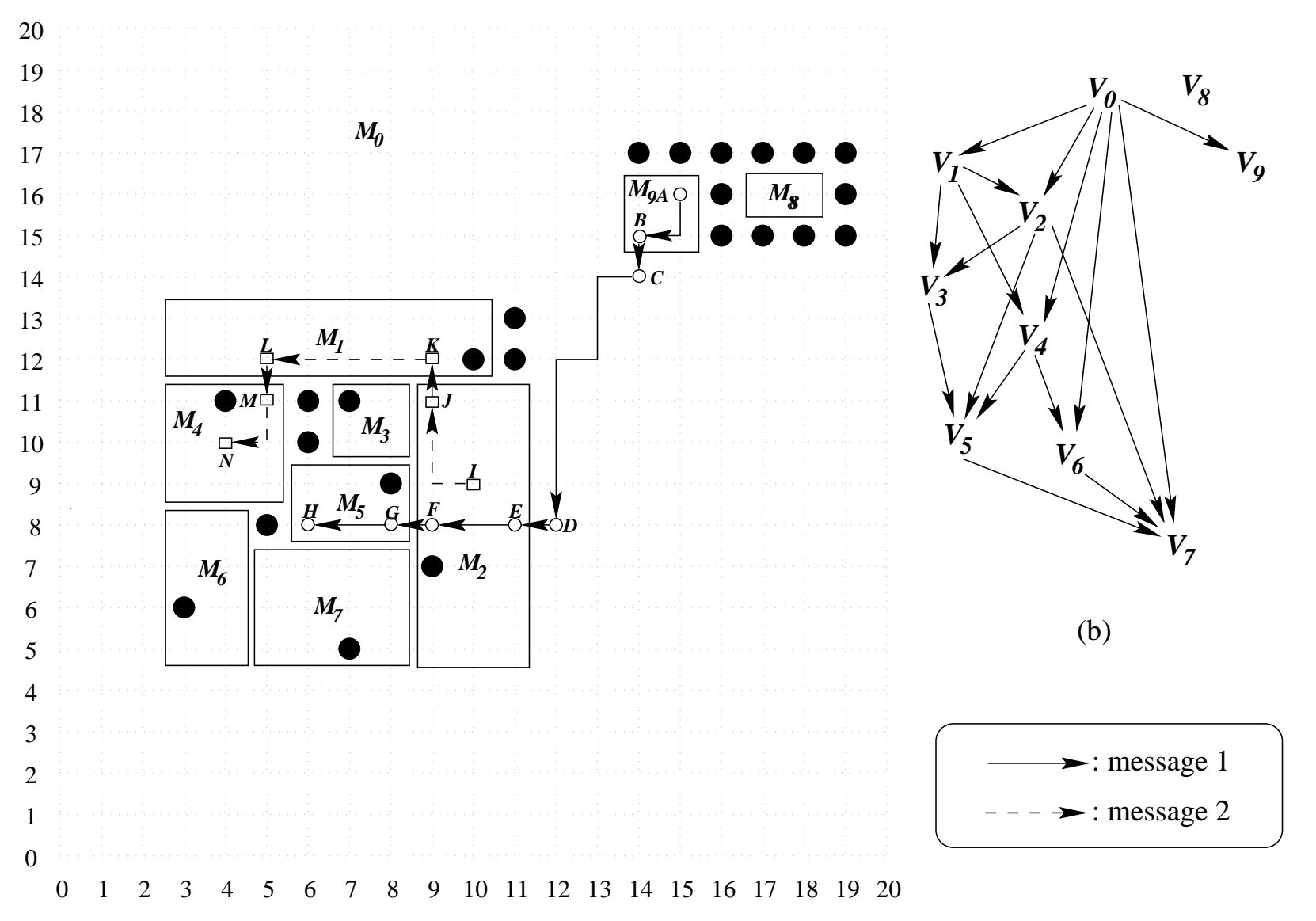

(a)

Figure 2. (a) shows a $21 \times 21$ injured mesh, where two messages 1 and 2 are routed by algorithm 3.1 . (b) shows the directed path graph constructed by algorithm 2.1.

2 Else if $\left(N_{C}=N_{S}\right)$ and $\left(S_{q}=S_{r}\right)$, then set header.dir to 1 or 2 .

3 Else if $\left(S_{q} \neq S_{r}\right)$, then update the message header by

(1) selecting one element of $T_{q, r}$, say $\left(w_{i}\right.$, dim, dir $)$, such that the sum of the distance between nodes $N_{C}$ and $w_{i}$ and the distance between nodes $w_{i}$ and $N_{D}$ is the smallest.

(2) setting header. $N_{I}$, header.dim, and header.dir to $w_{i}$, dim, and dir, respectively.

As shown in Figure 2(a), message 1 is routed from node $A(15,16)$ to node $H(6,8)$, and message 2 is routed from node $I(10,9)$ to node $N(4,10)$. For message 1 , the header is updated to $((6,8),(14,15),-1,2)$ due to step 3.1.3. Thus, it is routed to node $B(14,15)$ via $V I N_{2}$ by an adaptive deadlock-free routing algorithm in [6] due to step 3.3.2. Then it is routed to node $C(14,14)$ via $V C_{-1,2}$ due to step 2 . When message 1 reaches node $C$, the header is updated to $((6,8),(12,8),-0,1)$ due to step 3.1.3. Then it is routed to node $D(12,8)$ via $V I N_{1}$ and $V I N_{2}$ by an adaptive deadlock-free routing algorithm in [10] due to step 3.3.1, and is routed to node $E(11,8)$ via $V C_{-0,1}$ due to step 2. And so on, message 1 is routed to node $F(9,8)$ via $V I N_{1}$ [6] due to step 3.3.2, is routed to node $G(8,8)$ via $V C_{-0,1}$ due to step 2 , and is finally sent to node $H$ via $V I N_{1}$ by [6] due to step 3.2.2. For message 2, it is first routed to $J$ via $V I N_{2}$ by [6] due to step 3.3.2, is routed to node $K$ via $V C_{1,2}$ due to step 2 , is routed to node $L$ via $V I N_{1}$ by [6] due to step 3.3.2, is routed to node $M$ via $V C_{-1,1}$ due to step 2, is finally sent to node $N$ via $V I N_{1}$ by [6] due to step 3.2.2.

Theorem 1 Algorithm 3.1 is deadlock-free with two virtual channels per physical link. 


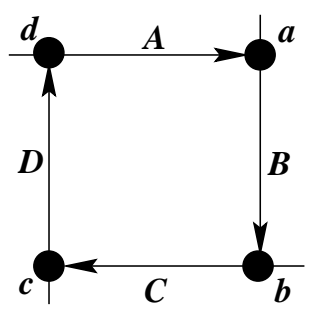

Figure 3. A waiting cycle of four messages $A, B, C$, and $D$.

PROOF. The proof is divided into three parts: (P1) we assign each virtual channel $x$ one channel number num $(x)$, (P2) we prove that a message can always find a virtual channel to use for each one hop in a non-decreasing order of channel numbers, (P3) A waiting cycle is not a real deadlock.

For P1, let virtual channel $x$ be output from a node in $S_{i}(i \geq 0)$. Then, $\operatorname{num}(x)$ is set to $i$ if virtual channel $x$ is in $V I N_{1}$, and $\operatorname{num}(x)$ is set to $-i$ if virtual channel $x$ is in $V I N_{2}$. For P2, suppose a message, whose destination $N_{D} \in S_{r}$, is sent to $N_{C}$ via virtual channel $y$ and will be sent out from $N_{C}$ via virtual channel $x$. We need to show $\operatorname{num}(x) \geq \operatorname{num}(y)$. If $N_{C} \neq N_{R}$, then $\operatorname{num}(x)=\operatorname{num}(y)$. Thus, we consider $N_{C} \in S_{q}$ receives the message from an intermediate node $N_{I} \in S_{t}\left(S_{t} \neq S_{q}\right)$ via virtual channel $y$. Three cases are discussed: (C2.1) vertex $V_{t}$ is an ancestor of vertex $V_{r}$ in the directed path graph, (C2.2) vertex $V_{r}$ is an ancestor of vertex $V_{t}$ in the directed path graph, and (C2.3) vertices $V_{r}$ and $V_{t}$ has least common ancestor $V_{l c a}$. For C2.1, $\left(V_{t}, V_{q}\right)$ is a directed edge in the directed shortest path $P_{\text {down }}$ from vertex $V_{t}$ to vertex $V_{r}$ due to step 4.1.2 of algorithm 2.1. It implies $t<q \leq r$ and virtual channels $x$ and $y$ are all in $V I N_{1}$. Thus num $(y)=t<q=\operatorname{num}(x)$. For C2.2, $\left(V_{q}, V_{t}\right)$ is a directed edge in the directed shortest path $P_{u p}$ from vertex $V_{r}$ to vertex $V_{t}$ due to step 4.1 .1 of algorithm 2.1. It implies $r \leq q<t$ and virtual channels $x$ and $y$ are all in $V I N_{2}$. And $\operatorname{num}(y)=-t<-q=\operatorname{num}(x)$. For C2.3, $\left(V_{q}, V_{t}\right)$ is a directed edge in the directed shortest path $P_{u p}$ from vertex $V_{l c a}$ to vertex $V_{t}$ due to step 4.1.3 of algorithm 2.1. If $V_{q}=V_{l c a}$, then virtual channel $y$ is in $V I N_{2}$ and virtual channel $x$ is in $V I N_{1}$. Thus, $\operatorname{num}(y)=-t<q=$ $\operatorname{num}(x)$. If $V_{q} \neq V_{l c a}$, then virtual channels $x$ and $y$ are all in $V I N_{2}$. And, num $(y)=-t<-q=\operatorname{num}(x)$. For P3, consider a waiting cycle as shown in Figure 3, where message $A$ (resp. $B, C, D$ ) holds virtual channel $d$ (resp. $a, b, c$ ) and requests $a$ (resp. $b, c, d$ ). By $\mathrm{P} 2$, we have $\operatorname{num}(a) \geq \operatorname{num}(d) \geq \operatorname{num}(c) \geq \operatorname{num}(b) \geq \operatorname{num}(a)$. It implies num $(a)=\operatorname{num}(d)=\operatorname{num}(c)=\operatorname{num}(b)$. Thus, virtual channels $a, b, c$ and $d$ are in a $M_{i}$. If $i=0$, then messages are routed by an adaptive and deadlock-free routing algorithm presented in [10]. Otherwise, messages are routed by an adaptive and deadlock-free routing algorithm presented in [6]. Thus, the waiting cycle is not a real deadlock.

\section{Conclusion}

In a concurrent multicomputer, a reliable routing algorithm requires deadlock-freedom and fault-tolerance. Many researchers $[1,3,10]$ proposed adaptive and deadlock-free routing algorithms using a certain number of virtual channels per physical link. But the shape of the tolerated faults should be rectangular. Thus, some good nodes need to be seen as faulty nodes and prohibited from interchanging messages with the other good nodes. In this paper, using two virtual channels per physical link, we propose an adaptive routing algorithm to tolerate irregular faulty patterns. Thus the node utilization is increased up to $100 \%$. Our method requires a central control unit or the global information of the node state.

\section{References}

[1] R. V. Boppana and S. Chalasani, "Fault-tolerant wormhole routing algorithms for mesh networks," IEEE Trans. Comput., vol. 44, pp. 848-864, 1995.

[2] Y. M. Boura and C. R. Das, "Fault-tolerant routing in mesh networks," International Conference on Parallel Processing, pp. 106-109, 1995.

[3] A. A. Chien and J. H. Kim, "Planar-adaptive routing: Lowcost adaptive networks for multiprocessors," 19th Annual International Symposium on Computer Arch., pp. 268-277, 1992.

[4] C. M. Cunningham and D. R. Avresky, "Fault-tolerant adaptive routing for two-dimensional meshes," 1st IEEE Symposium on High Performance Computer Arch., pp. 122-131, 1995.

[5] W. J. Dally and H. Aoki, "Deadlock-free adaptive routing in multicomputer networks using virtual channels," IEEE Trans. Parallel Distributed Syst., vol. 4., pp. 466-475, 1993.

[6] C. J. Glass and L. M. Ni, "Fault-tolerant wormhole routing in meshes," 23rd International Symposium on Fault-Tolerant Computing., pp. 240-249, 1993.

[7] R. L. Hadas and E. Brandt, "Original-based fault-tolerant routing in the mesh," 1st IEEE Symposium on High Performance Computer Arch., pp. 102-111, 1995.

[8] D. H. Linder and J. C. Harden, "An adaptive and faulttolerant wormhole routing strategy for $k$-ary $n$-cubes," IEEE Trans. Comput., vol. 40, pp. 2-12, 1991.

[9] C. Seitz et al., Wormhole Chip Project Report, Winter 1985. 
[10] C. C. Su and K. G. Shin, "Adaptive fault-tolerant deadlockfree routing in meshes and hypercubes," IEEE Trans. Comput., vol. 45, pp. 666-683, 1996.

[11] M. J. Tsai, "Adaptive Fault-Tolerant Routing for Multicomputers," submitted to IEEE Trans. Comput.. 